\title{
Magnetic imaging of ferromagnetic shape with Mobile Hall Sensor Array system
}

\begin{abstract}
The Mobile Hall Sensor Array system is a dynamic moving device capable of ferromagnetic shape evaluation based on the magnetic flux leakage testing principle. The device consists of a linearly integrated 1-D Hall Sensor array to detect magnetic field changes on a ferromagnetic shape under evaluation. The signal processing application software residing on a computer is developed for the Hall sensor array signals processing which leads to the magnetic image construction of magnetized ferromagnetic shape. This paper presents the principle of operation for Mobile Hall Sensor Array System in the shape detection of ferromagnetic materials. In addition, the signals array processing algorithm implemented in Hall Sensor Signals Array Processing application software is presented. The experimental results illustrated the magnetic image of specimens SS400 mild steel of various shape e.g square, round and triangle. The results proved the ability of Mobile Hall Sensor Array system to perform shape evaluation on ferromagnetic materials.
\end{abstract}

Keyword: Signal processing; Hall Sensor Array; Magnetic image 